\title{
Bases experimentais da utilização da cardiomioplastia no tratamento da insuficiência miocárdica.
}

Luiz Felipe P. MOREIRA*, Antônio C. P. CHAGAS*, Gustavo P. CAMARANO*, Idagene A. CESTARI*, Milton S. OSHIRO*, Eduardo NAKAYAMA*, Adolfo LEIRNER*, Protásio Lemos da LUZ*, Edgard A. LOPES*, Noedir A. G. STOLF*, Adib D. JATENE*.

MOREIRA, L. F. P.; CHAGAS, A. C. P.; CAMARANO, G. P.; CESTARI, I. A.; OSHIRO, M. S.; NAKAYAMA, E.; LEIRNER, A.; LUZ, P. L.; LOPES, E. A.; STOLF, N. A. G.; JATENE, A. D. - Bases experimentais da utilização da cardiomioplastia no tratamento da insuficiência miocárdica. Rev. Bras. Cir. Cardiovasc., 3(1): 9-20, 1988

RESUMO: A cardiomioplastia é uma técnica que utiliza enxertos musculares esqueléticos, estimulados sincronamente ao coração, para substituir, ou envolver o miocárdio. $O$ objetivo deste trabalho foi analisar as características contráteis e a resistência à fadiga do músculo grande dorsal normal e estimulado cronicamente, bem como avaliar a eficiência da cardiomioplastia como método de suporte circulatório. Treze cães foram estudados, após condicionamento elétrico do músculo grande dorsal esquerdo, por período de 6 semanas. Sete deles foram submetidos a medida isométrica da força exercida pelos músculos condicionados e pelos controles contralaterais e a estudo morfológico. Os parâmetros ideais de estimulação foram semeIhantes para os músculos normais e os condicionados. Os músculos condicionados, constituídos, predominantemente, de fibras de ação lenta, apresentaram uma força de amplitude menor $(-27 \%)$ e um tempo de contração mais longo $(+32 \%)$. Por outro lado, as curvas de fadiga dos músculos normais, constituídos de fibras mistas, mostraram a queda inicial da força de contração e valores estáveis, após 30 minutos, inversamente proporcionais à freqüência das contraçōes, resultando em um mesmo índice tensão-tempo (18 $\pm 2 \mathrm{kgF} . \mathrm{seg} / \mathrm{min})$. Já os músculos condicionados apresentaram um desempenho estável nas mesmas frequeências, mantendo um indice tensāo-tempo elevado $(68 \pm 6 \mathrm{kgF}$. $\varepsilon e \mathrm{~g} / \mathrm{min})$. Os outros 6 animais foram submetidos a cardiomioplastia, sendo estudados hemodinâmica e ecocardiograficamente, após a indução de disfunção miocárdica. Com a estimulação síncrona do músculo esquelético, observou-se a elevação do índice cardíaco em $36 \pm 4 \%$ ( $p<0,01)$, associada a queda da pressāo capilar pulmonar. $O$ aumento da fração de ejeção foi documentado $(51 \pm 3 \%)$, inclusive quando o enxerto foi colocado apenas sobre o ventrículo esquerdo $(p<0,01)$. Em conclusâo: 1$)$ os músculos esqueléticos são capazes de manter atividade semelhante ao trabalho cardíaco, com um desempenho que depende de sua capacidade aeróbica; 2) a estimulaçāo crônica leva à transformação adaptativa das fibras musculares, aumentando a sua capacidade de trabalho aeróbico; 3 ) a cardiomioplastia pode ser um método alternativo, no tratamento da insuficiência mioćardica, mesmo quando é possível apenas o envolvimento parcial do coração pelo enxerto.

DESCRITORES: cardiomioplastia; substituto miocárdico; suporte circulatório; miocárdio, insuficiência, cirurgia; estimulação muscular esquelética.

Trabalho realizado no Instituto do Coração do Hospital das Clínicas da Faculdade de Medicina da Universidade de Săo Paulo. São Paulo. SP. Brasil.

Apresentado ao 15: Congresso Nacional de Cirurgia Cardiaca. Rio de Janeiro, RJ, 8 e 9 de abril, 1988.

- Do Instituto do Coração do Hospital das Clínicas da Faculdade de Medicina da Universidade de Sảo Paulo.

Endereço para separatas: Luiz Felipe Moreira. Av. Dr. Enéas Carvalho de Aguir, 44. Divisão Cirúrgica. 05403 São Paulo, SP. Brasil. 
MOREIRA, L. F. P.; CHAGAS, A. C. P.; CAMARANO, G. P.; CESTARI, I. A.; OSHIRO, M. S.; NAKAYAMA, E.; LEIRNER, A.; LUZ, P. L.; LOPES, E. A.; STOLF, N. A. G.; JATENE, A. D. - Bases experimentais de utilização da cardiomioplastia no tratamento da insuficiência miocárdica. Rev. Bras. Cir. Cardiovasc., 3(1): 9-20, 1988.

\section{INTRODUÇÃO}

O tratamento de pacientes portadores de insuficiência miocárdica irreversivel ainda representa um grande desafio. Apesar dos recentes sucessos relatados com a utilização de vasodilatadores no controle da insuficiência cardiaca, a mortalidade de pacientes em classe funcional III e IV ainda é elevada ${ }^{32}$. Já os transplantes cardiacos enfrentam dificuldades na obtenção de doadores, necessitando, ainda, de rigoroso controle pós-operatório, por causa da terapêutica imunossupressora ${ }^{19}$, 21. Paralelamente, muitos progressos têm sido alcançados no desenvolvimento de dispositivos mecânicos de assistência circulatória, mas sāo relatados problemas como a obtenção de fontes de energia implantáveis e com a ocorrência de complicaçōes infecciosas e tromboembólicas ${ }^{34}$.

Por outro lado, muitos autores têm proposto a utilização de enxertos musculares esqueléticos pediculados, contraindo sincronamente ao coração, como nova alternativa terapêutica no tratamento da insuficiência miocárdica. $\mathrm{Na}$ cardiomioplastia, os enxertos musculares são utilizados para substituir ou envolver o miocárdio lesado ${ }^{10}, 12,15,25$, sendo relatados por CARPENTIER \&

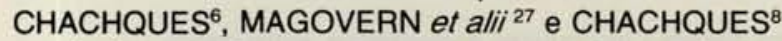
resultados promissores com o emprego dessa técnica no tratamento de grandes aneurismas do ventrículo esquerdo, da miocardiopatia isquêmica, ou dilatada e dos tumores ventriculares.

O emprego desse procedimento é limitado pela existência de poucos músculos que alcancem o mediastino mantendo seus pedículos intactos ${ }^{5}, 10,12,13,36$ e pela fadiga precoce dos músculos esqueléticos estimulados artificialmente $^{12,23}, 33,41,43$. Por outro lado, progressos significativos têm sido alcançados, no campo da estimulação muscular ${ }^{8}, 12,13,15,44$ e no estudo da transformação tecidual da musculatura esquelética por condicionamento elétrico, tornando-a resistente à fadiga $7,18,29,35,37$.

O objetivo deste trabalho foi analisar as características contráteis, a resistência à fadiga e as alterações morfológicas, decorrentes da estimulaçāo crônica do músculo grande dorsal, bem como avaliar a eficiência da cradiomioplastia como método de suporte circulatório em cães portadores de disfunção miocárdica induzida artificialmente, pelo uso de drogas.

\section{MATERIAL E MÉTODOS}

Treze cães mestiços, de peso variando entre 12 e $18 \mathrm{~kg}$, foram submetidos ao condicionamento elétrico do músculo grande dorsal esquerdo, por período de 6 a 8 semanas. Em 7 animais, foi analisada a força isométrica exercida pelos músculos condicionados e pelos controles contralaterais sob diferentes padrōes de estimulaçāo, sendo, também, realizado estudo anatomopa- tológico e histoquímico. Os outros 6 animais foram submetidos à cardiomioplastia, sendo avaliados hemodinâmica e ecocardiogaficamente, após a indução de disfunção miocárdica por bloqueio beta-adrenérgico e infusão de volume.

\section{Protocolo de Condicionamento Muscular}

Com o objetivo de se obter a transformação adaptativa das fibras do músculo grande dorsal por estimulação crônica, foi realizado o implante de eletrodos intramusculares e de um gerador de pulsos neurológico, modelo Itrel-Medtronic.

Após anestesia geral com pentobarbital sódico na dose de $30 \mathrm{mg}$ por $\mathrm{kg}$ de peso e entubaçāo orotraqueal, foi realizada a dissecção do músculo grande dorsal, através de uma incisão obliquaa na face lateral do hemitórax esquerdo. Foram mantidas as inserçōes tendíneas e o pedículo vásculo-nervoso principal do grande dorsal, procedendo-se, apenas, à interrupção da circulação colateral periférica. A seguir, dois eletrodos filamentares de aço, adaptados para conexão com o gerador de pulsos, foram implantados transversalmente, na face costal do músculo, mantendo uma distância de cerca de $6 \mathrm{~cm}$ entre si. O eletrodo do pólo negativo foi posicionado junto à ramificação proximal do nervo tóraco-dorsal e o gerador de pulsos foi implantado em uma loja na região abdominal.

A estimulação muscular foi iniciada uma semana após a operação, para permitir a adaptação do tecido muscular às alteraçōes da interrupção da circulação colateral. $\mathrm{O}$ grande dorsal foi estimulado com séries de pulsos com freqüência de $50 \mathrm{~Hz}$ e duração de $125 \mathrm{mseg}$, mantendo-se, ainda, uma amplitude de pulso adequada para permitir a contração efetiva do tecido muscular. Durante as 3 primeiras semanas de estimulação, o gerador de pulsos foi programado para provocar 60 contraçōes musculares por minuto e, nas semanas subseqüentes, a freqüência das contraçōes foi aumentada para 120 por minuto.

\section{Estudo da Força de Contração Muscular}

Para a realização desse estudo, foram especialmente desenvolvidos, pela Divisão de Bioengenharia do Instituto do Coração, um estimulador muscular com parâmetros variáveis e um sistema de fixação acoplado a um transdutor de força, para a avaliação da contraçāo isométrica do grande dorsal.

Sete animais foram estudados sob anestesia geral, após período de 6 a 8 semanas de estimulação muscular. O grande dorsal foi dissecado, mantendo-se o pedículo vascular ântero-superior e o nervo tóraco-dorsal intactos. Procedeu-se, então, à disenserção da aponeurose da região paravertebral e à fixação cio retalho muscular 
MOREIRA, L. F. P.; CHAGAS, A. C. P.; CAMARANO, G. P.; CESTARI, I. A.; OSHIRO, M. S.; NAKAYAMA, E.; LEIRNER, A.; LUZ, P. L.; LOPES, E. A.; STOLF, N. A. G.; JATENE, A. D. - Bases experimentais de utilização da cardiomioplastia no tratamento da insuficiência miocárdica. Rev. Bras. Cir. Cardiovasc., 3(1): 9-20, 1988

em um suporte metálico rígido, que foi conectado ao transdutor de força. Os estudos da força de contração isométrica dos músculos condicionados e do grande dorsal contralateral (grupo controle) foram realizados em dias separados. Foram analisados os resultados obtidos frente à variação da freqüência dos pulsos e da duração da série de pulsos, bem como da pré carga utilizada. Também foram determinados os limiares de estimulação e a resposta da força de contração à variação da amplitude e da largura dos pulsos de estimulação. Finalmente, a fadiga dos músculos normais e condicionados foi analisada, observando-se a queda da força de contração, sob diferentes freqüências de estimulação.

\section{Estudo Anatomopatológico e Histoquímico}

Com o objetivo de se analisarem as modificaçōes histológicas ocorridas nos tecidos musculares submetidos ao condicionamento elétrico, foram realizadas biópsias nos 3 segmentos do músculo grande dorsal, após o periodo de estimulação. Os fragmentos obtidos foram submetidos a microscopia óptica convencional, a microscopia eletrônica de transmissão em cortes ultrafinos e a método histoquímico para ATPase. Os músculos contralaterais também foram analisados, como grupo controle.

\section{Cardiomioplastia: Avaliação Hemodinâmica e Ecocardiográfica}

Após o período de condicionamento muscular, os outros 6 animais foram, novamente, anestesiados e submetidos a cardiomioplastia. O enxerto pediculado do músculo grande dorsal foi, nesse caso, introduzido no interior da cavidade torácica, através de uma janela obtida com a ressecção de cerca de $5 \mathrm{~cm}$ da porção anterior do segundo arco costal. O coração foi, a seguir, abordado através de uma toracotomia póstero-lateral esquerda, na altura do quinto espaço intercostal, sendo aberto o pericárdio longitudinalmente. Procedeu-se, entāo, ao envolvimento dos ventrículos direito e esquerdo, pelo retaIho muscular, que teve suas bordas superior e paravertebral fixadas na região do sulco atrioventricular (Figura 1). Ao final do procedimento, foi seccionada a inserção umeral do grande dorsal, sendo que, em 2 animais, foi realizado apenas o envolvimento do ventrículo esquerdo pelo enxerto muscular.

Os estudos hemodinâmico e ecocardiográfico foram realizados uma semana após a operação, com o animal anestesiado e com o tórax fechado. Foram medidos o débito cardíaco por termodiluição e as pressōes das câmaras direitas e esquerdas, concomitantemente à avaliação da função ventricular esquerda pela ecocardiografia uni e bidimensional. Após a realização das medidas em condição basal, sem a contração do enxerto muscular, foi induzida disfunção miocárdica. Utilizando-se um beta-

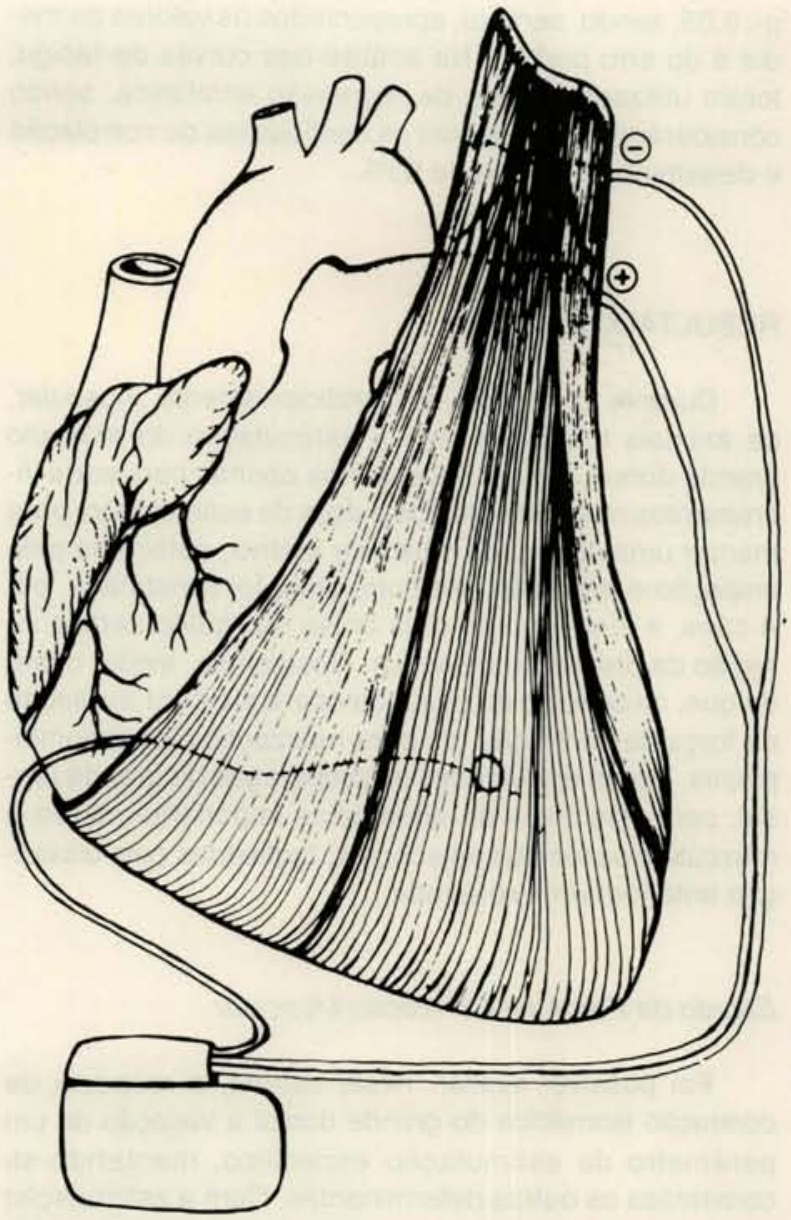

Fig. 1 - Esquema que mostra o enxerto pediculado do músculo grande dorsal envolvendo os ventrículos direito e esquerdo.

bloqueador endovenoso (Propranolol) na dose de $2 \mathrm{mg}$ por $\mathrm{kg}$ de peso e infusão de volume, procorou-se obter uma pressão capilar pulmonar acima de $15 \mathrm{~mm} \mathrm{Hg}$ e a queda do índice cardiaco no mínino em $30 \%$. Respeitando-se um intervalo de cerca de 15 minutos entre as medidas, para a estabilização da condiçāo circulatória, foram repetidas as avaliaçōes hemodinâmica e ecocardiográfica. Esses estudos foram realizados na presença de disfunção miocárdica, sem a contração do grande dorsal e durante a estimulação síncrona do enxerto muscular com a atividade ventricular.

\section{Análise Estatística}

Os resultados obtidos, nos estudos realizados com os músculos normais e condicionados, bem como os obtidos nos estudo do desempenho da cardiomioplastia após a indução de disfunçāo miocárdica, foram comparados entre si isoladamente, sendo analisados estatisticamente por meio do teste da diferença média de dados emparelhados, recorrendo-se à distribuição $T$ de Student. Fora considerados significativos os valores de 
MOREIRA, L. F. P.; CHAGAS, A. C. P.; CAMARANO, G. P.; CESTARI, I. A.; OSHIRO, M. S.; NAKAYAMA, E.; LEIRNER, A.: LUZ, P. L.; LOPES, E. A.; STOLF, N. A. G.; JATENE, A. D. - Bases experimentais de utilizaçāo da cardiomioplastia no tratamento da insuficiência miocárdica. Rev. Bras. Cir. Cardiovasc., 3(1): 9-20, 1988.

$\mathrm{p}<0,05$, sendo, sempre, apresentados os valores da média e do erro padrāo. $\mathrm{Na}$ análise das curvas de fadiga, foram utilizados testes de regressão estatística, sendo considerados significantes os coeficientes de correlação e determinação acima de $95 \%$.

\section{RESULTADOS}

Durante o período de condicionamento muscular, os animais toleraram bem a estimulação do músculo grande dorsal. Foram necessários apenas pequenos incrementos na amplitude dos pulsos de estimulação, para manter uma contração muscular efetiva, detectada pela inspeção e palpação. por outro lado, foi constatada, em 4 cães, a formação de uma bolsa de líqüido seroso na região da dissecção muscular. Destaca-se, ainda, o fato de que, na ocasião da segunda operaçāo para avaliação da força de contração, ou para realização da cardiomioplastia, houve dificuldades na preparação do grande dorsal, pela existência de aderências importantes entre o músculo e os tecidos adjacentes, motivadas pela dissecção anteriormente realizada.

\section{Estudo da Força de Contração Muscular}

Foi possivel avaliar, neste estudo, a resposta da contração isométrica do grande dorsal à variação de um parâmetro de estimulação específico, mantendo-se constantes os outros determinantes. Com a estimulação com séries de pulsos, observou-se a necessidade de uma freqüência acima de $25 \mathrm{~Hz}$ para a obtenção de uma contração uniforme dos músculos normais. Por outro lado, os músculos eletricamente condicionados contraíram uniformemente, a partir de $20 \mathrm{~Hz}$ de freqüência (Figura 2). Demonstrou-se, também, que os músculos submetidos à estimulação crônica apresentaram, com a freqüência de $50 \mathrm{~Hz}$, uma força de amplitude menor $(-27 \pm 5 \%)$ e um tempo de conração mais longo ( + $32 \neq 4 \%$ ) do que os músculos normais ( $<<0,04$ ). O Gráfico 1 mostra os resultados da amplitude da força de contraçāo obtidos com a variação da freqüência de pulsos. Os valores de força mais altos foram obtidos a partir de $50 \mathrm{~Hz}$, tanto para os músculos normais, como para os músculos condicionados. Já a análise da duração da série de pulsos mostrou que $150 \mathrm{mseg}$ foi o menor valor capaz de proporcionar uma contração de máxima amplitude nos estudos realizados. Adicionalmente, não foi observada qualquer influência da pré-carga, nos valores superiores a $0,5 \mathrm{KgF}$, sobre a contração isométrica dos músculos estudados.

A análise dos parâmetros eletrofisiológicos da estimulação muscular esquelética mostrou que, a partir do limiar de estimulação, a força de contração aumenta com a elevação da amplitude de pulso, até alcançar um patamar cujo pontc inicial é definido como limiar

\section{MÚSCULOS NÃO CONDICIONADOS}
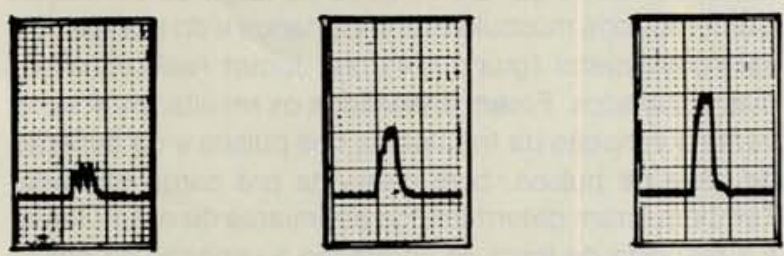

\section{MÚSCULOS CONDICIONADOS}

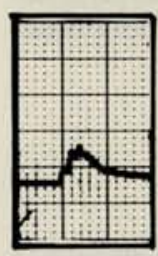

$10 \mathrm{~Hz}$

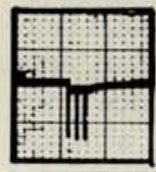

Fig. 2 - Curvas de contraçāo dos músculos esqueléticos normais e condicionados sob diferentes frequeências de estimulaçăo.

\section{GRÁFICO 1}

RESPOSTA DA AMPLITUDE DA FORÇA DE CONTRAÇĀO DOS MÚSCULOS ESQUELÉTICOSA VARIAÇĀO DE FREQÜENCIA DOS PULSOS DE ESTIMULAÇĀO

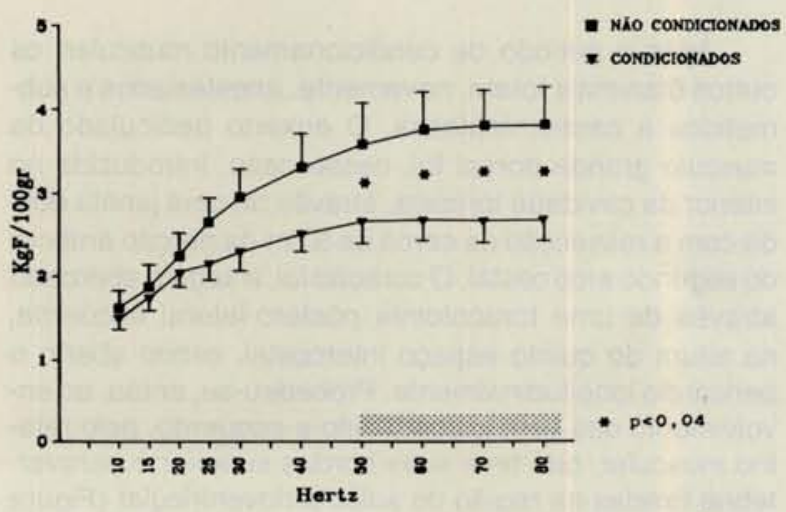

TABELA 1

PARAMETROS ELETOFISIOLÓGICOS DA ESTIMULAÇĀO MUSCULAR ESQUELÉTICA

NALORES MEDIDOS COM DURAÇĀO DE PULSO DE 0,6 MSEG)

\begin{tabular}{lll}
\hline & $\begin{array}{c}\text { Medidas } \\
\text { pós implante }\end{array}$ & $\begin{array}{c}\text { Medidas } \\
\text { crónicas }\end{array}$ \\
\hline Limiar de Estimulaçăo & $0,51 \pm 0.05$ volts $1,6 \pm 0,21$ Volts \\
\hline Limiar Força Máxima & $1,56 \pm 0,16$ Volts $3,86 \pm 0,47$ Volts \\
\hline Impedância & $242 \pm 21$ Ohms $168 \pm 13$ Ohms \\
\hline
\end{tabular}


MOREIRA, L. F. P.; CHAGAS, A. C. P.; CAMARANO, G. P.; CESTARI, I. A.; OSHIRO, M. S.; NAKAYAMA, E.; LEIRNER, A.; LUZ; P. L.; LOPES, E. A.; STOLF, N. A. G.; JATENE, A. D. - Bases experimentais de utilização da cardiomioplastia no tratamento da insuficiência miocárdica. Rev. Bras. Cir. Cardiovasc., 3(1): 9-20, 1988.

de força máxima. Na Tabela 1 , são apresentados os resultados dos limiares de estimulação e de força máxima, bem como os valores da impedância do sistema eletrodo - músculo esquelético. Estes dados foram obtidos logo após o implante dos eletrodos intramusculares em músculos normais, sendo, também, analisados os eletrodos cronicamente implantados nos músculos submetidos à estimulação por período de 6 a 8 semanas.

A queda percentual da amplitude da força de contração dos músculos normais e condicionados em relação ao tempo, com a freqüência de 75 contraçōes por minuto, é apresentada no Gráfico 2. O Gráfico 3 mostra a variação do índice tensāo-tempo, nas mesmas condiçōes de estimulação. Os valores esperados em 48 horas foram calculados, estatisticamente, por regressão geométrica, exibindo índices significantes de determinação e correlação. Adicionalmente, a resistência à fadiga dos músculos normais foi analisada sob diferentes freqüências de contração, tendo-se demonstrado que, após a queda inicial da força isométrica, são encontrados valores inversamente proporcionais à freqüência utilizada, resultando em um índice tensão-tempo semelhante cronicamente ( $\mathrm{Ta}$ -

\section{GRÁFICO 2}

QUEDA PERCETUAL DA FORÇA DE CONTRAÇÃO DO MÚSCULO GRANDE DORSAL NORMAL E CONDICIONADO EM RELAÇĀO AO TEMPO DE ESTIMULAÇĀO

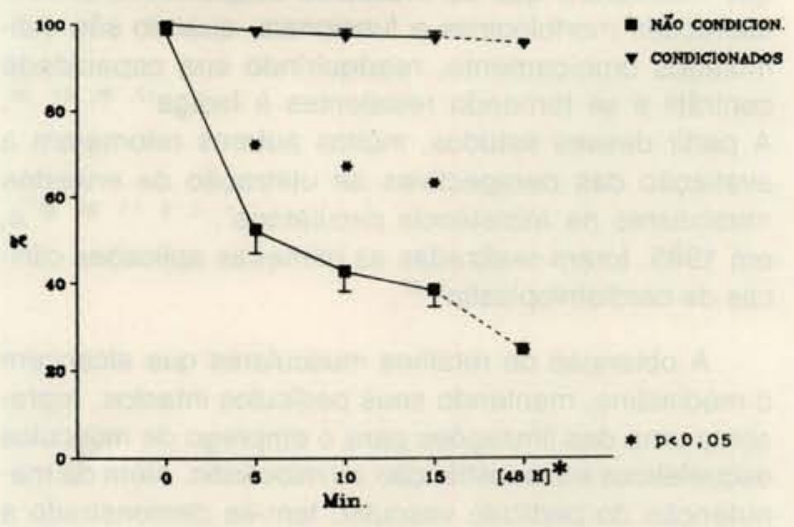

GRÁFICO 3

VARIACAAOO DO INDICE TENSÃO-TEMPO DO MÜSCULO

GRANDE DORSAL NORMAL E CONDICIONADO EM RELAÇĀO AO TEMPO DE ESTIMULAÇĀO

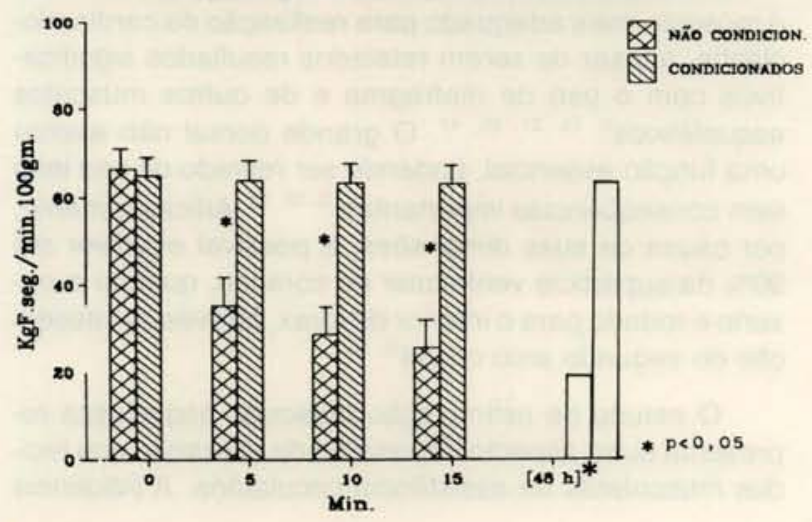

bela 2). Os músculos condicionados, quando analisados nas mesmas freqüências, apresentaram um desempenho estável, mantendo um indice tensão-tempo elevado $(68 \pm 6 \mathrm{KgF} . \mathrm{seg} / \mathrm{min})$.

TABELA 2

QUEDA PERCENTUAL DA FORCA DE CONTRACAÃOE VARIACCÃO DO INDICE TENSAO-TEMPO (I.T.T.) EM MUUSCULOS NORMAIS FRENTE A DIFERENTES FREQÜENCIAS DE CONTTRACÃO

\begin{tabular}{l|ccc}
\hline $\begin{array}{l}\text { Contraçōes } \\
\text { por min. }\end{array}$ & Tempo & $\begin{array}{c}\text { Valor Perc. } \\
\text { da Força }\end{array}$ & I.T.T. \\
\hline \multirow{4}{*}{25 c.p.m. } & Início & $100 \%$ & $24,7 \pm 2,5$ \\
\cline { 2 - 4 } & 15 min. & $76,8 \pm 7 \%$ & $19,1 \pm 2,6$ \\
\cline { 2 - 4 } & 48 horas & $69,5 \pm 2,1 \%$ & $17,8 \pm 0,4$ \\
\hline \multirow{4}{*}{37 c.p.m. } & Início & $100 \%$ & $35,4 \pm 3,2$ \\
\hline & 15 min. & $62,5 \pm 5 \%$ & $21,9 \pm 2,5$ \\
\cline { 2 - 4 } & 48 horas & $50,7 \pm 1,9 \%$ & $18,2 \pm 0,5$ \\
\hline \multirow{3}{*}{75 c.p.m. } & Início & $100 \%$ & $66,2 \pm 6,1$ \\
\cline { 2 - 4 } & 15 min. & $38,4 \pm 3,7 \%$ & $25,5 \pm 4,1$ \\
\cline { 2 - 4 } & 48 horas & $25,9 \pm 5,4 \%$ & $17,6 \pm 1,7$ \\
\hline
\end{tabular}

\section{Estudo Anatomopatológico e Histoquímico}

Não foram evidenciadas áreas de fibrose, ou de degeneração das fibras, pela microscopia óptica convencional, nos músculos submetidos ao condicionamento elétrico. O estudo histoquímico com ATPase demonstrou que os músculos ronicaente estimulados passam a se compor, uniformemente, de fibras lentas do tipo I, enquanto que os músculos normais apresentam os 2 tipos de fibras, com uma predominância de fibras rápidas do tipo II. Paralelamente, foi possível observar, pela microscopia eletrônica de transmissão, um maior número de mitocôndrias, associado a diminuição dos retículos sarcoplasmáticos, nas fibras dos músculos condicionados. A análise mais profunda e quantitativa das alteraçōes morfológicas provocadas pela estimulaçāo crônica dos músculos esqueléticos será motivo de um relato posterior.

Cardiomioplastia: Avaliaçāo Hemodinâmica e Ecocardiográfica

A análise do desempenho da cardiomioplastia como método de suporte circulatório demonstrou que essa técnica foi capaz de elevar o índice cardiaco, às custas do aumento do volume sistólico, em $36 \pm 4 \%$ na presença de disfunção miocárdica $(p<0,01)$. Observou-se, também, na mesma situação, queda da pressão capilar pulmonar (Gráfico 4), bem como diminuição das resistências vasculares. Por outro lado, as pressōes em câmaras direitas não se alteraram, significativamente, no modelo 
MOREIRA, L. F. P.; CHAGAS, A. C. P.; CAMARANO, G. P.; CESTARI, I. A.; OSHIRO, M. S.; NAKAYAMA, E.: LEIRNER, A. LUZ, P. L.; LOPES, E. A.; STOLF, N. A. G.; JATENE, A. D. - Bases experimentais de utilização da cardiomioplastia no tratamento da insuficiência miocárdica. Rev. Bras. Cir. Cardiovasc., 3(1): 9-20, 1988.

experimental utilizado, principalmente quando o enxerto muscular foi colocado apenas sobre o ventrículo esquerdo. A Tabela 3 mostra os resultados do estudo ecocardiográfico, sendo importante destacar que a elevação da fração de ejeção, durante a estimulação síncrona do grande dorsal (Figura 3), foi também observada nos casos em que foi realizado apenas o envolvimento parcial do coração pelo músculo esquelético.

\section{GRÁFICO 4}

DESEMPENHO HEMODINAMICO DA CARDIOMIOPLASTIA EM MODELO DE INSUFICIENNCIA MIOCARDICA

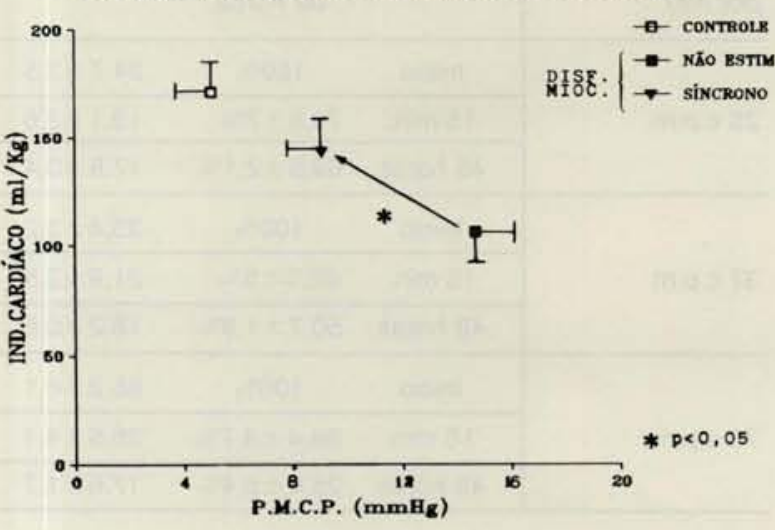

TABELA 3

INDICES ECOCARDIOGRAFICOS DO DESEMPENHO VENTRICULAR ESQUERDO EM CÄES SUBMETIDOS A CARDIOMIOPLASTIA

\begin{tabular}{lccc}
\hline & \multicolumn{3}{c}{ Disfunção Miocárdica } \\
\hline & $\begin{array}{c}\text { Controle pré } \\
\text { disfunção }\end{array}$ & $\begin{array}{c}\text { Enxerto não } \\
\text { estimulado }\end{array}$ & $\begin{array}{c}\text { Enxerto } \\
\text { sincronizado }\end{array}$ \\
\hline Variação diâmetro & $37 \pm 2,4 \%$ & $15,4 \pm 1,1 \%$ & $26,3 \pm 1,7 \%$ \\
\hline Fração de Ejeção & $74,6 \pm 2,8 \%$ & $39,3 \pm 2,4 \%$ & $59,6 \pm 3,9 \%$ \\
\hline
\end{tabular}

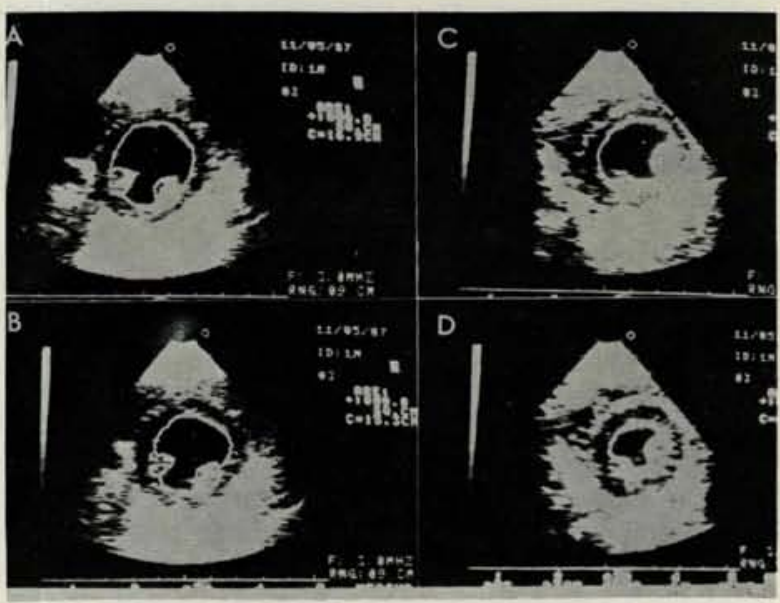

Fig. 3 - Ecocardiograma bidimensional que mostra a contraçăo ventricular esquerda, antes (A, B) e durante a estimulação sincrona do grande dorsal com a atividade cardiaca (C, D), na presença de disfunçăo miocárdica.

\section{DISCUSSĀO}

A utilização de enxertos musculares esqueléticos, estimulados sincronamente ao coração, em substituição ao miocárdio, ou como mecanismos de propulsão em dispositivos de assitência circulatória, tem sido proposta, por vários autores, como uma alternativa no tratamento da insuficiênci: miocárdica irreversivel.

Desde que LERICHE \& FONTAINE ${ }^{24}$, em 1933, descreveram o uso de enxertos livres de tecidos musculares, na correçāo de aneurismas do ventrículo esquerdo, muitos progressos têm sido relatados. KANTROWITZ \& Mc KINNON ${ }^{20}$ propuseram, em 1958, a utilizaçāo de marcapassos para estimular, sincronamente, músculos esqueléticos colocados sobre o coração, ou em volta da aorta, e coube a NAKAMURA \& GLENN ${ }^{33}, 5$ anos mais tarde, demonstrarem a elevação da pressão arterial com a contração do diafragma ao redor do coração. Estudos posteriores comprovaram a importância da estimulaçāo com séries de pulsos para obtenção de contraçōes musculares uniformes e mais potentes ${ }^{23.41,44}$, tendo-se observado, no entanto, existência de fadiga muscular, representada pela queda da força de contração do enxerto com a estimulação prolongada ${ }^{23}, 33,41,43$. Essa limitação foi contornada, por outro lado, a partir dos trabalhos que mostraram que os músculos esqueléticos sofrem alteraçōes morfológicas e funcionais, quando sāo estimulados cronicamente, readquirindo sua capacidade contrátil e se tornando resistentes à fadiga ${ }^{17}, 35,37,38$. A partir desses estudos, muitos autores retomaram a avaliação das perspectivas da utilização de enxertos musculares na assistência circulatória ${ }^{1}, 3.9 .11,26,28 \mathrm{e}$, em 1985, foram realizadas as primeiras aplicaōes clínicas da cardiomioplastia ${ }^{6,27}$.

A obtenção de retalhos musculares que alcancem o mediastino, mantendo seus pedículos intactos, representa uma das limitações para o emprego de músculos esqueléticos em substituição ao miocárdio. Além da manutenção do pedículo vascular, tem-se demonstrado a importância das terminações nervosas para preservação da arquitetura dos enxertos musculares ${ }^{11}, 33,35,36$, principalmente quando não são estimulados artificialmente ${ }^{39}$. ${ }^{40}$. Estudos anatômicos apontam o grande dorsal como o músculo mais adequado para realizaçāo da cardiomioplastia, apesar de serem relatados resultados significativos com o uso de diafragma e de outros músculos esqueléticos $^{5}, 15,21,25,41$. O grande dorsal nāo exerce uma função essencial, podendo ser retirado de seu leito sem conseqüências importantes ${ }^{10,12,13}$. Adicionalmente, por causa de suas dimensōes, é possível envolver até $90 \%$ da superfície ventricular do coração, quando o enxerto é rodado para o interior do tórax, através da ressecção do segundo arco costal ${ }^{8,10}$.

O estudo da estimulação muscular esquelética representa outro aspecto importante da utilização dos tecidos musculares na assistência circulatória. A influência 
MOREIRA, L. F. P.; CHAGAS, A. C. P.; CAMARANO, G. P.; CESTARI, I. A.; OSHIRO, M. S.: NAKAYAMA, E.; LEIRNER, A.; LUZ, P. L.; LOPES, E. A.; STOLF, N. A. G.; JATENE, A. D. - Bases experimentais de utilizaçāo da cardiomioplastia no tratamento da insuficiência miocárdica. Rev. Bras. Cir. Cardiovasc., 3(1): 9-20, 1988.

da estimulação com séries de pulsos sobre a contração muscular foi analisada, neste estudo, à semelhança de outros trabalhos da literatura ${ }^{7}, 11,22,23,41,44$. A obtenção de uma contração uniforme e de maior amplitude e duração com os músculos esqueléticos depende da fusão de pequenas contraçōes desencadeadas em seqüência, quando são utilizadas séries de estímulos com freqüências elevadas $^{7,44}$. O músculo grande dorsal normal exibe freqüências de fusão maiores do que os músculos cronicamente estimulados, fato explicado porque a contração dos músculos submetidos a longos períodos de estimulação torna-se mais lenta. Paralelamente, esses músculos exibem uma força de amplitude menor do que os músculos normais, também em conseqüência da transformaçāo que as fibras musculares sofrem com a estimulação artificial, passando de fibras rápidas e de maior amplitude de contração para fibras de contração lenta ${ }^{7}$ $11,35,38$. Os níveis de força apresentados pelo grande dorsal, depois do período de codicionamento elétrico, são, contudo, compativeis com o trabalho exercido pelo músculo cardíaco ${ }^{12,30}$. Finalmente, considerando-se os mínimos valores necesários para provocar uma contração de máxima amplitude como parâmetros ideais para estimulação da musculatura esquelética, observou-se, neste relato, que os melhores resultados foram obtidos com a freqüēncia de pulsos de $50 \mathrm{~Hz}$ e a duração da série de pulsos de $150 \mathrm{mseg}$, tanto para os músculos normais quanto para os músculos condicionados eletricamente. Os mesmos valores também têm sido apontados por outros autores, em estudos semelhantes ${ }^{11,44}$.

Paralelamente, ao analisar-se, neste estudo, os niveis de energia utilizados na estimulação da musculatura esquelética com eletrodos intramusculares, observou-se a necessidade de valores muito superiores ao limiar de estimulaçāo, para se obter a contração efetiva dos enxertos musculares. Os valores do limiar de força máxima foram ainda mais elevados, quando se analisararam os eletrodos implantados cronicamente, sendo importante destacar que esses dados, associados ao fato de que foram encontrados valores baixos de impedância nos mesmos eletrodos, refletem em um consumo de energia elevado para a obtenção de um desempenho adequado dos músculos esqueléticos. Outros autores também têm relatado resultados semelhantes ${ }^{12},{ }^{15}$, destacando-se, contudo, a necessidade de niveis menores de energia na estimulação muscular, quando os eletrodos são colocados diretamente sobre o nervo motor ${ }^{11,25}$. Esse tipo de estimulação é limitado, no entanto, pela possibilidade de se lesar o pedículo nervoso, levando à atrofia e à perda do retalho muscular ${ }^{39}$.

Outros fatores estão, ainda, envolvidos com a utilização de enxertos musculares esqueléticos na cardiomioplastia e em dispositivos de assistência circulatória. Para se iniciar a estimulação muscular, é necessário aguardar um período de adaptaçẫo vascular depois da rotação do retalho, pois a dissecção dos músculos esqueléticos leva à interrupção da circulação colateral e à isquemia transitória do enxerto, nas duas semanas subseqüen- tes $^{31}$. A ocorrência de necrose em enxertos musculares estimulados precocemente pode ser uma conseqüência deste fato $^{28}$. Limitados pela fadiga, na fase inicial da estimulação, os músculos esqueléticos passam, a seguir, por uma fase de transformação morfológica e funcional das fibras, que dura cerca de 6 semanas $^{7}, 17,18$, 35,37 . A musculatura esquelética é constituída de fibras dos tipos I e II, mas o condicionamento elétrico leva à transformação das fibras do tipo II, que sāo rápidas e sensíveis à fadiga, em fibras do tipo I, responsáveis por uma contração lenta e resistentes à fadiga ${ }^{7}, 29.35$, 37. Essa alteração é acompanhada, a nivel celular, da elevação do número de mitocôndrias, da diminuição dos retículos sarcoplasmáticos e de alteraçōes na estrutura da banda $Z^{17}$. É importante ressaltar, ainda, que ocorrem mudanças, no conteúdo enzimático e na composição da miosina, com a estimulação elétrica e que as células musculares passam de um metabolismo predominantemente glicolítico para uma atividade quase que puramente oxidativa ${ }^{18,29,35}$. O reflexo dessas mudanças é a otimização do desempenho muscular, sendo possível manter-se, cronicamente, um trabalho efetivo com um consumo de oxigênio reduzido ${ }^{2}$.

O grau de transformação sofrido pelas células musculares, bem como a resistência à fadiga dos músculos condicionados eletricamente, varia proporcionalmente ao tipo de estimulação utilizada durante a fase de condicionamento, o que reflete a existência de um mecanismo adaptativo ${ }^{29}, 35,42$. Por outro lado, observou-se, neste relato, que a queda da amplitude da força exibida pelos músculos normais varia inversamente à periodicidade das contraçōes musculares, o que resulta em um mesmo trabalho desempenhado cronicamente, sob diferentes freqüências de contração. Esses dados, obtidos pela análise do índice tensão-tempo, mostram que a fadiga representa uma diminuição parcial de capacidade contrátil dos músculos esqueléticos, refletindo, provavelmente, a perda de função das fibras glicolíticas do tecido muscular. Adicionalmente, estudos realizados em fases diferentes do condicionamento elétrico mostram que a transformação das fibras musculares leva à elevação progressiva da resistência à fadiga, simultaneamente à ocorrēncia de alteraçōes morfológicas que implicam na elevaçāo da capacidade aeróbica dos músculos esqueléticos $^{18}$. Finalmente, tem sido sugerido por alguns autores que a estimulação síncrona dos enxertos, com a atividade cardíaca em ciclos alternados, pode minimizar o problema da fadiga inicial ${ }^{8,17}$ e que os músculos esqueléticos podem se transformar, enquanto exercem sua função no sítio da assistência circulatória'.

O desempenho de ventrículos artificiais, impulsionados por enxertos musculares estimulados eletricamente, também tem sido estudado por vários autores. Existem evidências de que essas bombas musculares, quando conectadas a circuitos hidráulicos, ou à própria circulação, são capazes de gerar pressōes e fluxos compativeis com o trabalho cardíaco por períodos prolonga- 
MOREIRA, L. F. P.; CHAGAS, A. C. P.; CAMARANO, G. P.; CESTARI, I. A.; OSHIRO, M. S.; NAKAYAMA, E.; LEIRNER, A.: LUZ, P. L.; LOPES, E. A.; STOLF, N. A. G.; JATENE, A. D. - Bases experimentais de utilização da cardiomioplastia no tratamento da insuficiência miocárdica. Rev. Bras. Cir. Cardiovasc., 3(1): 9-20, 1988.

$\operatorname{dos}^{1}, 3,4,30$. Outros estudos destacam que ventrículos moldados com o músculo grande dorsal podem exercer trabalho com uma potência intermediária à dos ventrículos direito e esquerdo ${ }^{28}$, sendo, ainda, demonstrado que os músculos esqueléticos podem ser, também, influenciados por um mecanismo do tipo Frank-Starling ${ }^{4}$. ${ }^{22}$. Este fato, porém, não foi comprovado com os valores de pré carga utilizados neste estudo, em ensaio isométrico de força. Finalmente, têm sido relatados benefícios hemodinâmicos com a utilização de enxertos musculares em bombas de contrapulsação extra-aórtica ${ }^{11,}{ }^{28}$, ou conectadas em série na aorta, ou na pulmonar ${ }^{1,26}$. A limitaçāo desses métodos na assistência circulatória, por outro lado, deve-se a uma incidência ainda elevada de fenômenos tromboembólicos ${ }^{1.26}$.

Paralelamente, publicaçōes recentes têm ressaltado as possibilidades da utilização da cardiomioplastia no tratamento dos aneurismas e tumores do ventrículo esquerdo, da miocardiopatia isquêmica ou dilatada e de anomalias congênitas com hipofunção do coração direito ${ }^{5,8,12}, 26$. Nesta técnica, a colocação de enxertos musculares esqueléticos sobre a superfície do miocárdio leva à formação de aderências e de uma intensa circulação colateral entre os tecidos, principalmente, nos casos em que se faz a escarificação do epicárdio. Este fato, além de propiciar uma interação entre os tecidos envolvidos durante a contração síncrona dos mesmos, pode ser importante como mecanismo de revascularização, tanto do miocárdio como do músculo esquelético ${ }^{5}, 10,13$. Por outro lado, nos casos em que se processa a substituição do miocárdio pelo enxerto muscular, tem sido descrita uma alta incidência de trombos nas cavidades ventriculares ${ }^{16,40}$, sendo importante o revestimento interno do enxerto com um tecido biológico estabilizado, como o pericárdio autógeno tratado previamente com glutaraldeído ${ }^{8,16}$. Deve-se ressaltar, também, a importância da orientação das fibras dos músculos esqueléticos utilizados na cardiomioplastia, para a obtenção de um auxílio efetivo à sistole ventricular, sendo salientado, por outros autores, que a colocação das fibras do enxerto, paralelamente ao septo interventricular, pode levar a um encurtamento melhor do diâmetro ântero-superior do ventrículo esquerdo $^{14}$. Em estudos experimentais, tem sido descrita a manutençăo de um débito cardíaco normal, com substituição parcial do miocárdio dos ventrículos direito ou esquerdo pelo músculo grande dorsal, sendo demonstrado que a contração dos enxertos musculares foi responsável, nos 2 casos, por um aumento significativo do volume sistólico e da fração de ejeção ${ }^{9}$. Paralelamente, neste estudo, procurou-se estudar o desempenho do músculo grande dorsal colocado sobre o coraçāo em um modelo de insuficiência cardíaca, sendo, também, observado o aumento significativo da fração de ejeção do ventrículo esquerdo e do débito cardíaco, associado à queda das pressōes venosas, com a contraçāo síncrona do enxerto muscular na presença de disfunção miocárdica. A experiência clínica inicial com a utilização da cardiomioplastia, por outro lado, não tem apresentado resultados significantes. As indicaçōes dessa técnica foram feitas principalmente em pacientes onde se associaram outros procedientos, como ressecção de tumores e aneurismas do ventrículo esquerdo ${ }^{6,8,27}$. Adicionalmente, foram utilizados, em alguns casos, sistemas inadequados de estimulação, o que torna ainda mais dificil uma análise efetiva dos resultados apresentados ${ }^{8,}{ }^{27}$. Destaca-se, porém, a documentação ao aumento da fração de ejeção do ventrículo esquerdo, em todos os pacientes estudados durante a estimulação síncrona do enxerto muscular, fato que se torna mais evidente principalmente durante o exercício ${ }^{6,8,27}$. Finalmente, a indicaçāo da cardiomioplastia em pacientes com miocardiopatias dilatadas, principalmente as de origem chagásica, enfrenta um problema adicional que é o fato de não se conseguir um envolvimento adequado do coração pelo enxerto na presença de grandes cardiomegalias, sendo esta informação retirada de um estudo anatômico ainda não publicado. Nos 2 experimentos deste estudo, em que foi realizado apenas o envolvimento parcial do coração pelo grande dorsal, nāo foram observadas, por outro lado, diferenças significativas nos resultados obtidos em relação aos casos submetidos a cardiomioplastia total.

As conclusōes deste estudo foram: 1) os músculos esqueléticos, quando submetidos a estimulação elétrica com séries de pulsos, apresentam características contráteis semelhantes às do miocárdio; 2) o desempenho crônico dos enxertos musculares utilizados na assistência circulatória depende de sua capacidade de trabalho aeróbico; 3) a estimulação prolongada leva à transformação adaptativa das fibras musculares, aumentando seu metabolismo aeróbico, podendo este fato ocorrer com o enxerto contraindo sincronamente ao coração, enquanto desempenha sua função de auxílio circulatório; 4) a cardiomioplasta pode ser um método alternativo no tratamento da insuficiência miocárdica irreversivel, inclusive em pacientes com grande cardiomegalia, onde seja possível apenas o envolvimento parcial do coração pelo enxerto muscular. Finalmente, a utilização de enxertos musculares esqueléticos, na assistência circulatória e na cardiomioplastia, abre novas perspectivas para o tratamento da insuficiência miocárdica, sendo, contudo, importante a realização de pesquisa clínica criteriosa para a real comprovação de sua eficácia. 
MOREIRA, L. F. P.; CHAGAS, A. C. P.; CAMARANO, G. P.; CESTARI, I. A.; OSHIRO, M. S.; NAKAYAMA, E.; LEIRNER, A.: LUZ, P. L.; LOPES, E. A.; STOLF, N. A. G.; JATENE, A. D. - Bases experimentais de utilização da cardiomioplastia no tratamento da insuficiência miocárdica. Rev. Bras. Cir. Cardiovasc., 3(1): 9-20, 1988.

MOREIRA, L. F. P.; CHAGAS, A. C. P.; CAMARGO, G. P.; CESTARI, I. A.; OSHIRO, M. S.; NAKAYAMA, E.; LEIRNER, A.; LUZ, P. L.; LOPES, E. A.; STOLF, N. A. G.; JATENE, A. D. - Experimental basis of cardiomyoplasty utilization in the treatment of myocardial insufficiency. Rev. Bras. Cir. Cardiovasc. 3(1): 9-20, 1988.

ABSTRACT: Beneficial effects of cardiomyoplasty have been documented and the use of this technique in the treatment of dilated cardiomyopathy have been suggested. This study was undertaken to evaluate the contracting and fatigue characteristics of normal and conditioned Latissimus Dorsi and the effectiveness of stimulated preconditioned skeletal muscle flaps wrapped around the heart to restore ventricular contractility in presence of myocardial dysfunction. Thirteen adult mongrel dogs were studied after burst stimulation of left Latissimus Dosi for six to eight weeks. In seven animals, conditioned muscles were compared with unconditioned contralateral controls by isometric force development. Normal and transformed muscles exhibited the same optimum pacing parameters (burst frequencies from $50 \mathrm{~Hz}$ up, train duration from 150 ms up). Conditioned muscles showed a smaller force $(-27 \%)$ and a longer contraction time $(+32 \%)$. Fatigue curves of unconditioned muscles under different duty cycles showed marked initial decrease and inversely proportional stable values of force after some minutes with equal final tension time indexes (18 $\pm 2 \mathrm{KgF}$. $\mathrm{seg} / \mathrm{min})$. Conditioned Latissimus Dorsi maintained stable force during prolonged stimulation under same conditions with a greater tension time index $(68 \pm 6 \mathrm{KgF}$. seg/min). The other six animals were submited to cardiomyoplasty; hemodynamic and echocardiographic evaluation were performed after induction of myocardial dysfunction by betablockers administration and volume ioading. With synchronous pulse train stimulation increases of cardiac index by an average of $36 \pm 4 \%(p<0.01)$ were observed, associated with a decrease of pulmonary wedge pressure. Ejection fraction augmentation of $51 \pm 3 \%$ above the control was also documented in same conditions $(p<0.01)$, even when muscle flap was wrapped only around the left ventricle. In conclusion, this study reveals that skeletal muscles are capable to maintain steady-state work similarly to the myocardium with a power dependent of their aerobic capacity. It also suggests the occurrency of muscle adaptive transformation, increasing its steady-state work capacity, with chronic pulse train stimulation. In addition, cardiomyoplasty may be an alternative method of treatment for irreversible cardiomyopathy, including when muscle flap may wrapp only partially the heart.

DESCRIPTORS: cardiomyoplasty; circulatory support; myocardial insufficiency, surgery; skeletal muscle stimulation.

\section{REFERÊNCIAS BIBLIOGRÁFICAS}

1 ACKER, M. A.; ANDERSON, W. A.; HAMMOND, R. L.; CHIN, A. J.; BUCHANAN, J. W.; MORSE, C. C.; KELLY, A. M.; STEPHENSON, L. W. - Skeletal muscle ventricles in circulation: one to eleven weeks' experience. J. Thorac. Cardiovasc. Surg., 94(2): 163-174, 1987.

2 ACKER, M. A.; ANDERSON, W. A.; HAMMOND, R. L.; DiMEO Jr., F.; MC CULLUM, J.; STAUM, M.; VELCHIK, M.; BROWN, W. E.; GALE, D.; SALMONS, S.; STEPHENSON, L. W. - Oxygen consumption of chronically stimulated skeletal muscle. J. Thorac. Cardiovasc. Surg., 94(5): 702-709, 1987.

3 ACKER, M. A.; HAMMOND, R. L.; MANNION, J. D.; SALMONS, S.; STEPHENSON, L. W. - An autologous biologic pump motor. J. Thorac. Cardiovas. Surg., 92(4): 733-746, 1986.

4 ACKER, M. A.; HAMMOND, R. L.; MANNION, J. D.; SALMONS, S.; STEPHENSON, L. W. - Skeletal muscle as the potential power source for a cardiovascular pump: assessment in vivo. Science, 236(4799): 324-327, 1987.
5 APPIANI, E.; ALMADA, H.; GARLANDO, C.; MILET, J.; BASTAROLI, J. - Potential myocardial revascularization and mechanical effects of pectoral muscle transplant. Ann. Plast. Surg., 10(3): 252-263, 1983.

6 CARPENTIER, A. \& CHACHQUES, J. C. - Myocardial substitution with a stimulated skeletal muscle: first successful clinical case. Lancet, 2(8440): 1267, 1985. (Resumo).

7 CARPENTIER, A.; CHACHQUES, J.C.; GRANDJEAN, P.; PERIER, P.; MITZ, V.; BOURGEOIS, I. - Transformation dún muscle squelettique par stimulation sequentielle progressive en vue de son utilisation comme substitut myocardique. C. R. Acad. Sc. Paris, 301(Série III, n: 11): 581-586, 1985.

8 CHACHQUES, J. C. - Cardiomioplastia: reconstruccion anatomica y funcional del corazon. Rev. Fed. Arg. Cardiol., 16(3): 185-193, 1987.

9 CHACHQUES, J. C.; GRANDJEAN, P. A.; MIHAILEANU, S.; BOURGEOIS, I.; CARPENTIER, A. - Dynamic cardiomyoplasty: a surgical approach to restore ventricular contractility. In: BELHASSEN, B.; FELDMAN, S.; COPPERMAN, Y. Cardiac pacing and electrophysiology. Tel Aviv, Israel, 1987. p. 531-534. 
MOREIRA, L. F. P.; CHAGAS, A. C. P.; CAMARANO, G. P.; CESTARI, I. A.; OSHIRO, M. S.; NAKAYAMA, E.; LEIRNER, A.: LUZ, P. L.; LOPES, E. A.; STOLF, N. A. G.; JATENE, A. D. - Bases experimentais de utilização da cardiomioplastia no tratamento da insuficiência miocárdica. Rev. Bras. Cir. Cardiovasc., 3(1): 9-20, 1988.

CHACHQUES, J. C.; MITZ, V.; HERO, M.; ARHAN, P.; GALLIX, P.; FONTALIRAN, F.; VILAIN, R. - Experimental cardioplasty using the latissimus dorsi muscle flap. J. CArdiovasc. Surg., 26(5): 457-462, 1985.

11 CHIU, R. C. J.; WALSH, G. L.; DEWAR, M. L.; DE SIMON, J. H.; KHALAFALLA, A. S.; IANUZZO, D. - Implantable extra-aortic baloon assist powered by transformed fatigue-resistant skeletal muscle. J. Thorac. Cardiovasc. Surg., 94(5): 964-701, 1987.

KOCHAMBA, G. \& CHIU, R. C. J. - The physiologic characteristics of transformed skeletal muscle for cardiac assist. Trans. Am. Soc. Artif. Intern. Organs, 33(3): 404-407, 1987
23 KUSABA, E.; SCHRAUT, W.; SAWATANI, S.; JARON, D.: FREED, P.; KANTROWITZ, A. - A diaphragmatic graft for augmenting left ventricular function: a feasibility study. Trans. Am. Soc. Artif. Intern. Organs. 19 251-257. 1973

24 LERICHE, R. \& FONTAINE, R. - Essai experimentale de traitement de certains infarctus du myocarde et de I anévrisme du coeur par une graffe du muscle strié. Bull. Soc. Nat. Chir., 59: 229-232, 1933.

25 MACOVIAK, J. A.; STEPHENSON, L. W.; SPIELMAN, S.; GREENSPAN, A.; LIKOFF, M.; SUTTON, M. S. J.; REICHEK, N.; RASHKIND, W. J.; EDMUNDS Jr., L. H. - Replacement of ventricular myocardium with diaphragmatic skeletal muscle: short-term studies. J. Thorac. Cardiovas. Surg., 81(4): 519-527, 1981.

26 MACOVIAK, J. A.; STINSON, E. B.; STARKEY, T. D.; HANSEN, D. E.; CAHILL, P. D.; MILLER, D. G.; SHUMWAY, N. E. - Myoventriculoplasty and neoventricle myograft cardiac augmentation to establish pulmonary blood flow: preliminary observations and feasibility studies. J. Thorac. Cardiovasc. Surg., 93(2): 212-220, 1987.

27 MAGOVERN, G. J.; HECKLER, F. R.; PARK, S. B.; CHRISTLIEB, I. Y.; MAGOVERN Jr., G. J.; KAO, R. L.; BENCKART, D. H.; TULLIS, G.; ROZAR, E.; LIEBLER, G. A.; BURKHOLDER, J. A.; MAHER, T. D. - Paced latissimus dorsi used for dynamic cardiomyoplasty of left ventricular aneurysms. Ann. Thorac. Surg., 44(4): $379-388,1987$.

28 MANNION, J. D.; ACKER, M. A.; HAMMOND, R. L.; FALTEMEYER, W.; DUCKETT, S.; STEPHENSON, L. W. - Power output of skeletal muscle ventricles in circulation: short-term studies. Circulation, 76(1): 155-163. 1987.

29 MANNION, J. D.; BITTO, T.; HAMMOND, R. L.; RUBINSTEIN, N. A.; STEPHENSON, L. W. - Histochemical and fatigue characteristics of conditioned canine latissimus dorsi muscle. Circ. Res., 58(2): 298-304, 1986.

30 MANNION, J. D.; HAMMOND, R.; STEPHENSON, L. W - Hydraulic pouches of canine latissimus dorsi: potential for left ventricular assistance. $J$. Thorac. Cardiovas. Surg., 91(4): 534-544, 1986.

31 MANNION, J. D.; VELCHIK, M. A.; ACKER, M. A.; HAMMOND, R.; ALAVA, A.; STEPHENSON, L. W. - Transmural blood flow of multi-layered latissimus dorsi skeletal muscle ventricles during circulatory assistance. Trans. Am. Soc. Artif. Intern. Organs, 32: 454-460, 1986.

32 MASSIE, B. M. \& CONWAY, M. - Survival of patients with congestive heart failure: past, present and future prospects. Circulation, 75(Supl. 4): 11-19, 1987.

33 NAKAMURA, K. \& GLENN, W. L. - Graft of the diaphragm as a functioning substitute for the myocardium. J. Surg. Res., 4 (3): 435-439, 1964. 
MOREIRA, L. F. P.; ChAGAS, A. C. P.; CAMARANO, G. P.; CESTARI, I. A.; OSHIRO, M. S.; NAKAYAMA, E.; LeIRNER, A.; LUZ, P. L.; LOPES, E. A.; STOLF, N. A. G.; JATENE, A. D. - Bases experimentais de utilização da cardiomioplastia no tratamento da insuficiência miocárdica. Rev. Bras. Cir. Cardiovasc., 3(1): 9-20, 1988.

34 PAE, W. E. \& PIERCE, W. S. - Combined registry for the clinical use of mechanical ventricular assist. Pumps and the total artificial hearts: first official report. J. Heart Transpl., 6(2): 68-70, 1987.

35 PETTE, D. \& VRBOVÁ, G. - Neutral control of phenotypic expression in mammalian muscle fibers. Muscle Nerve, 8(8): 676-689, 1985.

36 PHILLIPS, W. L.; PALLIN, S.; CRASTNOPOL, P. - Diaphragm transplantation: a preliminary report on an experimental form of permanent myocardial assistance. Angiology, 20(11): 628-933, 1969.

37 SALMONS, S. \& SRÉTER, F. A. - Significance of impulse activity in the transformation of skeletal muscle type. Nature, 263(5572): 30-34, 1976.

38 SALMONS, S. \& VRBOVÁ, G. - The influence of activity on some contractile characteristics of mammalian fast and slow muscles. J. Physiol., 201(3): 535-540, 1969. $c$

39 SHEPHERD, M. P.; TAMAKI, H.; MUSTARD, W. T. - Experimental study of the paced denervated diaphragmatic pedicle graft. Br. J. Surg., 55(2): 91-92, 1968.

40 SOLA, O. M.; DILLARD, D. H.; IVEY, T. D.; HANEDA, K.; ITOH, T.; THOMAS, R. - Autotransplantation of skeletal muscle into myocardium. Circulation, 71(2): 341-348, 1985.

41 SPOTNITZ, H. M.; MERKER, C.; MALM, J. R. - Applied physiology of the canine rectus abdominis: forcelength curves correlated with functional characteristics of a rectus powered "ventricle" potencial for cardiac assistance. Trans. Am. Soc. Artif. Intern. Organs, 20(Pt. B): 747-756, 1974.

42 SRETER, F. A.; PINTER, K.; JOLESZ, F.; MABUCHI, K. - Fast to slow transformation of fast muscles in response to longterm phasic stimulation. Exp. Neurol., 75(1): 95-102, 1982.

43 TERMET, H.; CHALENCON, J. L.; ESTOUR, F. - Transplantation sur le myocarde d'un muscle strié excité par pacemaker. Ann. Chir. Thorac. Cardiovas. (Paris), 5(2): 260-263, 1966.

44 VACHON, B. R.; KUNOV, H.; ZINGG, W. - Mechanical properties of diaphragm muscle in dogs. Med. Biol. Eng., 13(3): 252-260, 1975.

\section{Discussão}

\section{Dr. DIMINGO BRAILE \\ São José do Rio Preto, SP}

Acho que o trabalho do Luiz Felipe demonstra toda a capacidade que ele tem, e que a Instituição Incór também tem. É muito importante, que, aqui no Brasil, não fiquemos tão distantes da tecnologia que existe em outros países. Como todos sabem, este trabalho foi desenvolvido na França, e o Luiz Felipe, eu e mais o Noedir
Stolf estivemos, recentemente, lá e, na realidade, ele está numa fase ainda muito experimental e os casos clínicos que têm não são, absolutamente, comparáveis, porque a técnica é muito inicial, foi começada em pacientes com diferentes patologias. Existe uma dificuldade grande, ainda, de comparar um paciente com outro. Este trabalho desenvolvido pelo Luiz Felipe é absolutamente pioneiro e ele demonstrou coisas que ainda nāo tinham sido demonstradas. (Slide) Nós trabalhamos com um animal alterativo, que foi o carneiro, e temos procurado estudar uma outra faceta deste tipo de técnica, bastante semelhante, Aqui está o músculo grande dorsal e, aqui, o coração. (Slide) Aqui, detalhes técnicos de como envolvemos o coração (slide) e, aqui, está o coração, totalmente envolvido. (Slide) O cárdio-estimulador que nós desenvolvemos já tem uma freqüēncia padronizada de $30 \mathrm{~Hz}$, que é a freqüência que parece ser a melhor e nós podemos mudar o interpulso e a voltagem. Com isto, conseguimos fazer um aparelho um pouco menor, que pode ser deixado junto com o animal (slide), de tal forma que pode ficar preso ao animal e podemos ter esse animal andando, correndo e sendo avaliado posteriormente. (Slide) Aqui está a expressão do funcionamento do aparelho. Aqui está um QRS do próprio carneiro e este QRS deflagra o número de pulsos que fica, então, na fase sistólica da contração miocárdica. (Slide) Numa velocidade maior, é possível ver o QRS e a estimulação do músculo esquelético. (Slide) Aqui está a peça de um desses carneiros, depois de um mês de estimulação; vejam o coração e o músculo envolvendo-o. (Slide) Tirando o músculo, vejam como fica sobre o coração, Existe uma forte aderência do músculo ao coração e isto é necessário para que o procedimento funcione. (Slide) Aqui é um corte, onde se vêem o músculo cardiaco e o músculo esquelético que envolve o coração. Vejam, em maior aproximação, que existe, realmente, uma interface muito interessante e o músculo esquelético, realmente, parece que se soma ao músculo cardíaco (Slide). Aqui está a histologia e esta é a interface e este é o lugar que estamos estudando com maior cuidado, porque a interface entre o músculo esquelético e o músculo cardíaco tem que ser estudada e nos temos dedicado a isto. É lógico que existe uma transformação das fibras. (Slide) Aqui está a fibra esquelética. Acredito que é muito importante que este trabalho seja desenvolvido no nosso país, pois não sei até que ponto isto tenha aplicaçāo clínica direta. Ainda persistem dúvidas sobre a diferença da eficácia, quando se cobre um só ventrículo, ou se cobrem os dois. De qualquer forma, a transformação de energia muscular em um outro tipo de energia, por exemplo: carregando uma bateria, ou, mesmo, impulsionando uma câmara com válvulas, poderia ser uma alterantiva para o uso deste procedimento. Eu acredito que a demonstração que o Luiz Felipe fez tão bem, mostrando que, realmente, o músculo esquelético se transforma praticamente num músculo cardiaco, que tem a sua capacidade de contração e que mantém um trabaIho efetivo a longo prazo, vai fazer com que nós possamos continuar nesta linha de pesquisa em vários cam- 
MOREIRA, L. F. P.; CHAGAS, A. C. P.; CAMARANO, G. P.; CESTARI, I. A.; OSHIRO, M. S.; NAKAYAMA, E.; LEIRNER, A.; LUZ, P. L.; LOPES, E. A.; STOLF, N. A. G.; JATENE, A. D. - Bases experimentais de utilização da cardiomioplastia no tratamento da insuficiência miocárdica. Rev. Bras. Cir. Cardiovasc., 3(1): 9-20, 1988.

pos, não só envolvendo o coração, desenvolvendo outros tipos de mecanismos com os quais possamos usar essa energia desenvolvida dentro do próprio organismo. Novamente, desejo parabenizar o Luiz Felipe, o grupo do Incór; realmente, o trabalho é monumental. Para que vocês saibam, ele apresentou este trabalho para o Professor Carpentier e o impressionou fortemente, bem como seu grupo que existe em Paris, principalmente pela qualidade do trabalho, pelo desempenho e pela seriedade com que ele foi feito, pela contribuição que vem sendo dada por este trabalho, mostrando que, realmente, as fibras tipo 1 podem ser estimuladas já desde o inicio. Esta era uma outra dúvida séria que se tinha: se era necessário, primeiramente, treinar o músculo para, depois, transportá-lo para o coração. Hoje. se sabe que pode transplantar, esperar a simples aderância do músculo; ele já começa com um programa de treinamento do músculo, que, dentro de um período relativamente curto, vai ter a sua função ativada. Muito obrigado.

\section{DR. LUIZ FERNANDO KUBRUSLY Rio de Janeiro, RJ}

Eu gostaria de parabenizar os autores, em especial ao Dr. Luiz Felipe, pelo brilhante trabalho. Já estava difícil de fazer o comentário, antes de o Dr. Braile falar; mas, agora, ficou quase impossivel, mas a experiência deste tipo de procedimento é restrita, são poucos os Centros de pesquisa. Gostaria de me deter, simplesmente, a algumas aplicaçōes clínicas, que, eventualmente, a cardiomioplastia venha ter. (Slide). O Dr. Luiz Felipe referiu-se à indicação como método alternativo no tratamento da insuficiência miocárdica; eu acho que a dúvida vem quando esse método pode ser usado no paciente, se esse paciente é aquele em que, num determinado momento, nós vamos indicar ou um transplante cardíaco, ou a cardiomioplastia. Eu acho que esse procedimento deve ser usado antes da fase que o doente vá ao transplante cardíaco, porque ele vai ser submetido a uma cirurgia de grande porte, ele vai ter que sair vivo da cirurgia e ter que se sustentar hemodinamicamente por um periodo de, pelo menos, 20 a 30 dias, até que esse pedículo tenha fibrose, a interface, como diz o Dr. Braile, com o coração, mas não só com o coração, mas com o saco pericárdico. Eu acredito que a indicação talvez mais precisa vai ser com pacientes com contra-indicação para transplante cardiaco. (Slide) O segundo aspecto que gostaria de comentar é quanto à tècnica, no que diz respeito ao condicionamento físico. O Dr. Braile acabou de falar, também, do condicionamento muscular prévio, que, na aplicação clínica, está passando a ser desnecessário, em virtude do fato de que somos obrigados a esperar de 15 a 20 dias para se obter parte do resultado hemodinâmico do pedículo; então, já que se espera esse período, pode-se fazer a estimulação desse músculo e a transformação histoquímica dele, num músculo tipo 1 , com processo oxidativo, sem produção de ácido lático. Outros aspectos que eu deixaria de lado seriam a incisāo, a espera, como nós falamos, e a dificuldade na obtenção desse marcapasso, a que o $\mathrm{Dr}$. Luiz Felipe se referiu, em termos do tipo de marcapasso que vai ser necessário para esse procedimento. Muito obrigado.

\section{Dr. MOREIRA \\ (Encerrando)}

Gostaria, inicialmente, de agradecer ao Dr. Braile e ao Dr. Kubrusly, pelos comentários elogiosos. O Dr. Braile iniciou, praticamente conosco, o estudo desse tipo de cirurgia e, várias vezes, juntos, tivemos a oportunidade de discutir, amplamente, o problema. Ele passou a trabalhar, sob o ponto de vista que nós, infelizmente, nâo tivemos condiçōes de realizar, que foi sobre a interface do músculo, principalmente, estimulando cronicamente esses animais. Esse estudo da interface cronicamente, é um estudo bastante importante, pois talvez apresente, hoje, a principal limitação para a utilização da técnica. Como o Dr. Kubrusly também comentou, hoje se abandonou o condicionamento prévio da musculatura esquelética como idéia, principalmente porque é necessária, praticamente obrigatória, a espera de duas semanas, a partir da cirurgia, para que se obtenha uma aderência desse enxerto ao coração, ao pericárdio, bem como a sua revascularização, a sua adaptação vascular, a ligadura de todas as suas colaterais; isto, praticamente, representa maior limitaçāo, uma vez que, sem dúvida, podemos, a partir da segunda semana, já começar com a estimulação, obtendo, praticamente, 1/3 da potência que o nosso enxerto será capaz de desenvolver. Ainda com respeito ao comentário do $\mathrm{Dr}$. Kubrusly, quanto às indicações, restringi, realmente, as indicaçōes a pacientes que não sejam terminais, havendo, porém, a chance de se associar a cardiomioplastia, conforme uma colocação do Professor Jatene, a métodos de assistência circulatória durante essa fase inicial. Um outro detalhe, em relaçāo à cardiomioplastia dilatada: a grande cardiomegalia, realmente o ventrículo direito, pode ser uma das limitaçōes da aplicaçāo dessa técnica, razão pela qual também devemos olhar com mais critério os pacientes, numa fase mais precoce, quando com resistência pulmonar elevada, ou ventrículo direito bastante aumentado; o não envolvimento do ventrículo direito realmente poderá representar uma melhora parcial, porém a longo prazo não efetivo. Finalmente, o Dr. Braile colocou a possibilidade da utilzação desses enxertos na assistência circulatória, num senso mais global, talvez como bombas conectadas a nível circulatório. Muitos autores também já têm estudado essa possibilidade, sendo, porém, a limitação, aí, somada às da cardiomioplastia, uma limitação a mais que é a possibilidade de tromboembolismo ainda nesse tipo de aparelhos. Para concluir, eu acredito que a estimulação da musculatura esquelética, a cardiomioplastia, está, hoje, na sua fase inicial, dando seus primeiros passos, talvez possa representar, no futuro, uma alternativa efetiva para o tratamento de pacientes com indicação para transplante cardiaco. Muito obrigado. 